\title{
STUDY OF HIBISCUS ROSASINENSIS AS A FEEDSTOCK FOR NATURAL COLORANT TO FOOD
}

\author{
Azmain A. Akash, Fahim R. Choudhury, and Iqbal Hossain* \\ Department of Chemical Engineering, Bangladesh University of Engineering \& Technology, Dhaka \\ 1000, Bangladesh.
}

\begin{abstract}
The application of coloring to food is widespread around the globe. Some of the artificial food colorants are highly carcinogenic. Exploration of new natural colorants has therefore been under research. The present study was aimed to evaluate Hibiscus rosasinensis flower as a potential feedstock for natural colorant. Crude anthocyanin was first extracted from Hibiscus rosasinensis flower using a Soxhlet apparatus with ethanol as the solvent. Flower to solvent ratio was found to affect the extraction largely. A ratio of 1:20 was found to give the highest fraction of extract. The cytotoxicity of crude anthocyanin extract was subsequently determined by Brine Shrimp Lethality Test. The $\mathrm{LC}_{50}$ value for crude anthocyanin extract was $2332 \mu \mathrm{g} / \mathrm{ml}$, which is higher than the required minimum threshold value. Hence the findings of present study would be useful towards the application of $H$. rosasinensis flower as a feedstock for natural colorant to food.
\end{abstract}

Keywords: Natural Colorant, Hibiscus rosasinensis, Soxhlet Apparatus, Ethanol, Cytotoxicity, Brine Shrimp Lethality Test,

\section{Introduction}

In recent times, the use of artificial coloring in food items is quite widespread. Since some synthetic colours have been found to have played a vital role in the causation of cancer in laboratory animals, the search for alternative, safe natural food colours has intensified and continues to top the priority lists of many research laboratories around the world [1].

An organic source would be safer and healthier as a source of food grade color. Such a source is Hibiscus rosasinensis, which is commonly known as China Rose. It is found abundantly in Bangladesh and other tropical countries. It is a well-known fact that the petals of China Rose are rich in anthocyanins, which are water soluble colouring pigments responsible for the colouring of the fruits and flowers.

Anthocyanins are natural coloring pigments belonging to the flavonoid family of compounds [2]. Anthocyanins are present in most fruits and vegetables such as grapes, apples, cherries, pomegranates, red onions, red cabbage, and red berries (blueberries, black currants, raspberries, and strawberries. Anthocyanins possess antidiabetic, anticancer, antiinflammatory, antimicrobial, and anti-obesity effects, as well as prevention of cardiovascular diseases (CVDs) [3].

Anthocyanins are extracted from plants as a crude mixture [4]. Solid-liquid extraction (SLE) is the classic approach which is used in recovering anthocyanins. Classical solvent extraction and Soxhlet extraction had been in use for a long time. More recent technologically sophisticated techniques are pursued to maximize the recovery of anthocyanin and minimize the amount of solvent and decrease the degradation or the alteration of the natural state of colorants. These includes microwave assistance extraction [5], ultrasound assisted extraction [6], supercritical extraction [7] and so on.

Prior to commercial use of any natural colour in the food industry, it is necessary to conduct several tests according to the standards of Food and Drug Administration to be assured about the non-toxic behavior of the colour. These tests include cytotoxicity test, acute toxicity test, chronic toxicity test, etc.

Researches had been carried out on anthocyanin extraction from flowers belonging to the genus Hibiscus, mostly $H$. sabdariffa and $H$. rosasinensis. Microwave assisted extraction of anthocyanin from $\mathrm{H}$. rosasinensis has been reported [8]. The Soxhlet extraction was also employed in an experiment to study the dying properties of the crude [9]. The effect of extraction time, solvent proportion and temperature during the Ultrasonic extraction of anthocyanin from H. sabdariffa was also studied [10]. However, no such studies were found that studies the effect of different extraction parameters on the extraction of anthocyanin from Hibiscus rosasinensis. To fill this research gap, this study was carried out.

So, the primary objectives of this study was to conduct the Soxhlet extraction of anthocyanin from $H$. rosasinensis using pure ethanol solvent and to analyse 
effects of solute-solvent ratio on extraction efficiency. Furthermore, the cytotoxicity of the crude extract was evaluated by measuring $\mathrm{LC}_{50}$ value using Brine Shrimp Lethality Test.

\section{Experimentation}

\subsection{Sample preparation}

Fresh Hibiscus rosasinensis (China Rose) flowers were picked up from local market. In order to ensure the sample used was from the same source throughout the experiments, the fresh sample was collected in sufficient quantities in two batches. The flowers were then washed with distilled water. Then these flowers were dried at $40^{\circ} \mathrm{C}$ temperature in an electric oven for 24 hours. The dried sample was crushed with mortars and pestles and reduced to fine particles. The crushed sample was refrigerated in a glass container at $4^{\circ} \mathrm{C}$ until further experimentations.

\subsection{Soxhlet extraction}

A weighed amount of dried sample was taken in the thimble and placed inside the extraction chamber shown in Fig. 1. A certain amount of ethanol was poured in the round bottle extraction flask, placed on the heating mantle so that the solute-solvent ratio was $1: 10,1: 20$ and 1:30, respectively for three different observations. Lastly, the condenser was placed on top of the extraction flask and all these parts were fixed vertically. The extraction was carried out for four hours for all observations.

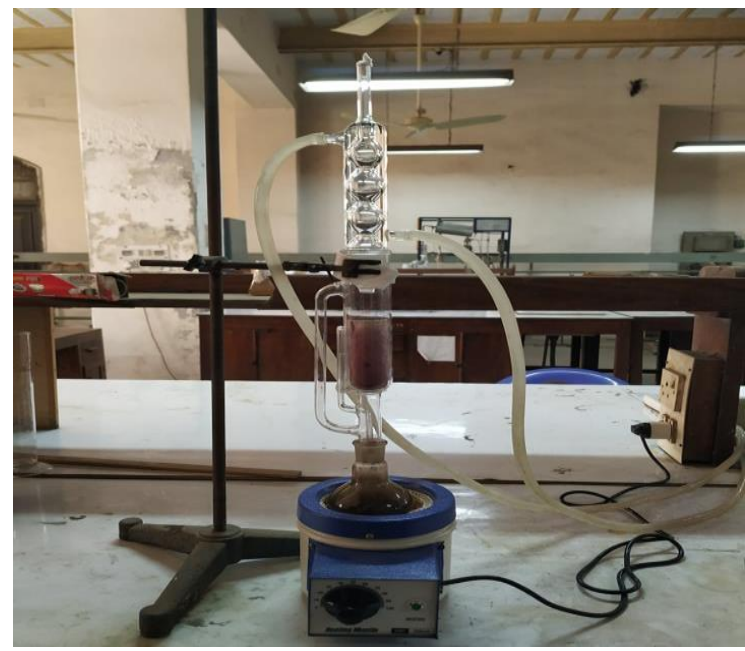

Fig. 1: Soxhlet extraction of $H$. rosasinensis

The separation of the crude anthocyanin from the ethanol solvent was then conducted through distillation and it was carried out until the solvent was fully recovered in the conical flask. The remaining crude extract was in the round bottle and it was weighed. The experimental setup used for separation is shown in Fig. 2. The extract was then collected in test-tube and refrigerated.

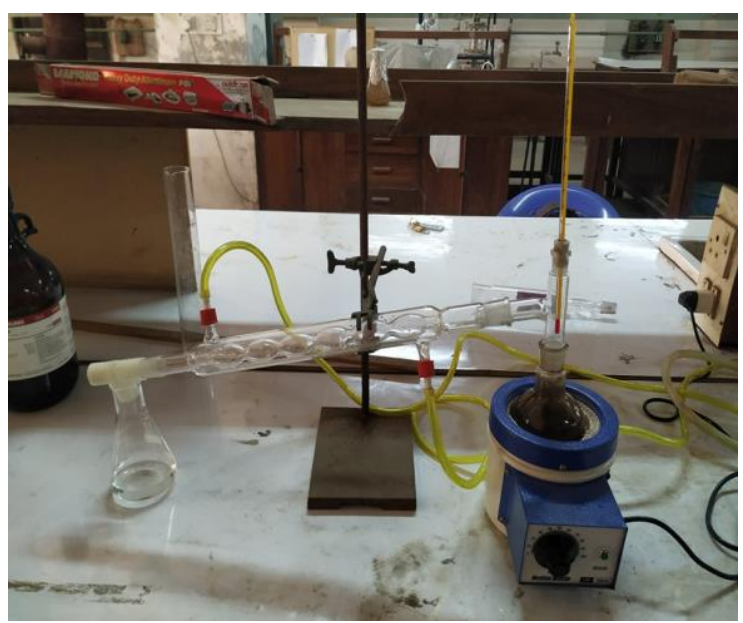

Fig. 2: Separation of ethanol and anthocyanin by bulb-type condenser

\subsection{Brine shrimp lethality test}

Preparation of seawater: Thirty eight (38) g sea salt (without iodine) was weighed, dissolved in one liter of distilled water and filtered off to get clear solution.

Hatching of brian shrimp: Artemia salina leach (brine shrimp eggs) collected from pet shops was used as the test organism. Seawater was taken in the small tank and shrimp eggs were added to one side of the tank and then this side was covered. Two days were allowed to hatch the shrimp and to be matured enough for experimentation. Constant oxygen supply was maintained through the hatching time. The hatched shrimps are attracted to the light (phototaxis) and so matured shrimp free from egg shell was collected from the illuminated part of the tank. These were taken from the fish tank by a pipette and diluted in fresh clear sea water to increase visibility and kept for further experimentation.

Preparation of test solutions: Sixty four (64) mg of crude extract was taken and dissolved in $200 \mu$ l of pure dimethyl sulfoxide (DMSO) and finally the volume was made to $20 \mathrm{ml}$ with seawater. Thus the concentration of the stock solution was $3200 \mu \mathrm{g} / \mathrm{ml}$.

The cytotoxic activity was subsequently performed by slight modification of the brine shrimp lethality bioassay method [11]. The stock solution at different concentrations obtained by serial dilution method were added to test tubes containing 10 shrimps in $5 \mathrm{ml}$ of prepared seawater to obtain the final concentrations of $1600,800,400,200,100,50,25,12.5,6.25$ and $3.125 \mu \mathrm{g} / \mathrm{ml}$, respectively. Each of these test solutions were incubated at room temperature. After 24 hours, the test tubes were inspected using a magnifying glass against a black background and the number of survived shrimps in each tube was counted. Shrimps 
were considered dead if they were lying immobile at the bottom of the vials. From this data, the percent (\%) of lethality of the brine shrimp was calculated for each concentration. The median Lethal Concentration $\mathrm{LC}_{50}$ of the test samples was determined by linear regression method from a plot of percentage mortality of shrimp against the logarithm of the sample concentrations. Dimethyl Sulfoxide (DMSO) was used as a negative control. Control groups are used in cytotoxicity study to validate the test method and ensure that the results obtained were only due to the activity of the test agent.

\section{Results \& discussions}

3.1 Comparison of extraction efficiency of crude anthocyanin from $H$. rosasinensis

Anthocyanin was extracted in two separate batches by Soxhlet extraction with three different solutesolvent ratios. The extraction efficiencies for are presented in Table 1 for the flowers of batch 1 and batch 2 respectively. The comparison is also shown as a bar chart in Fig. 3 for better visualization and understanding. Extraction efficiency is defined as follows-

Extraction efficiency $=$ (Mass of Anthocyanin extracted / Mass of dried flower used for the extraction) $\mathrm{x} 100 \%$

Table 1

Extraction efficiency of crude Anthocyanin for $H$. rosasinensis

\begin{tabular}{cccccc}
\hline \multirow{3}{*}{ Batch } & Solute- & Mass of & Volume of & Mass of & Extraction \\
& Solvent & dried & Ethanol \\
Ratio & lower $(\mathrm{g})$ & $(\mathrm{ml})$ & & xtract $(\mathrm{g})$ & $\begin{array}{c}\text { Efficiency } \\
(\%)\end{array}$ \\
\hline
\end{tabular}

\begin{tabular}{cccccc}
\hline & & & & & \\
& $1: 10$ & 15.67 & 156.80 & 0.484 & 3.090 \\
& $1: 20$ & 7.87 & 157.4 & 0.296 & 3.767 \\
& $1: 30$ & 8.36 & 250.5 & 0.295 & 3.520 \\
& $1: 10$ & 15.70 & 157.0 & 0.488 & 3.11 \\
& $1: 20$ & 13.27 & 265.4 & 0.492 & 3.73 \\
& $1: 30$ & 9.58 & 287.4 & 0.334 & 3.49 \\
\hline
\end{tabular}

Extraction efficiency was found in the range of 3.09 -3.77 for this study. Same trend and behavior were observed for both batches of flowers. It was found highest for the solute-solvent ratio of 20 for the three ratios considered in this study. When the ratio was increased from 10 to 20 , the extraction efficiency increased to a relatively higher value. This is probably due to the reason that potential for more extraction existed and the added proportion of solvent subsequently caused more extraction resulting in higher extraction efficiency. When the solute-solvent ratio was further increased from 20 to 30 , the extraction efficiency dropped to a slightly lower value. It is assumed that the added solvent introduced resistant to the extraction process lowering the efficiency. However, this observation clearly indicates that there is an optimum solute-solvent ratio and the ratio is always required to be chosen carefully for reasonable extraction efficiency.

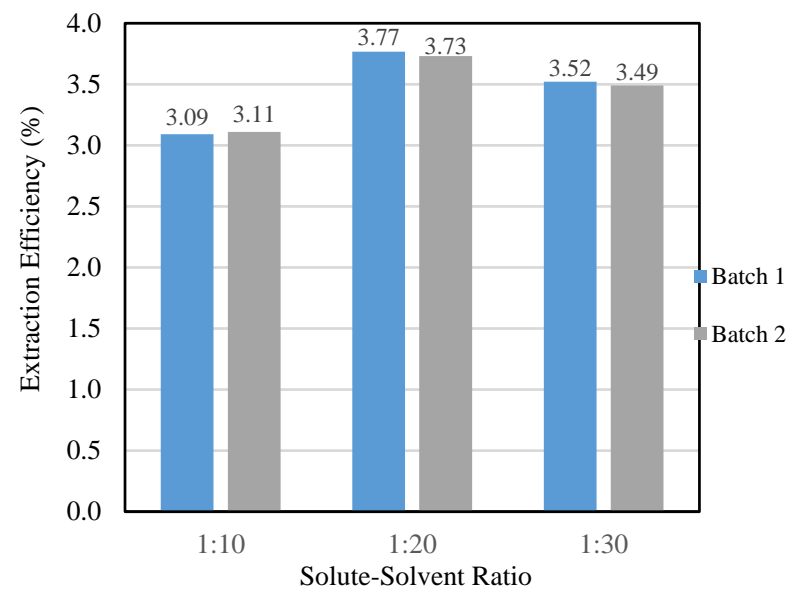

Fig. 3: Comparison of extraction efficiency for different solutesolvent ratio

\subsection{Evaluation of the cytotoxic activity of flower} extracts

According to Meyer's toxicity index, extracts with $\mathrm{LC}_{50}<1000 \mu \mathrm{g} / \mathrm{ml}$ are considered as toxic, while extracts with $\mathrm{LC}_{50}>1000 \mu \mathrm{g} / \mathrm{ml}$ are considered as nontoxic. [11]

Table 2

Cytotoxic activity of $H$. rosasinensis extract

\begin{tabular}{ccccc}
\hline No. & $\begin{array}{c}\text { Concentration, C } \\
(\mu \mathrm{g} / \mathrm{ml})\end{array}$ & $\log \mathrm{C}$ & $\begin{array}{c}\% \text { of } \\
\text { Mortality }\end{array}$ & $\begin{array}{c}\mathrm{LC}_{50} \\
(\mu \mathrm{g} / \mathrm{ml})\end{array}$ \\
\hline 1 & 1600 & 3.20412 & 47 & \\
2 & 800 & 2.90309 & 40 & \\
3 & 400 & 2.60206 & 37 & \\
4 & 200 & 2.30103 & 31 & \\
5 & 100 & 2 & 23 & 2332 \\
6 & 50 & 1.69897 & 20 & \\
7 & 25 & 1.39794 & 15 & \\
8 & 12.5 & 1.09691 & 0 & \\
9 & 6.25 & 0.79588 & 0 & \\
\hline
\end{tabular}

From the experiment, it can be observed that degree of mortality varied with exposure to different concentration levels of the test samples. The degree of mortality was found to be directly proportional to the concentration ranging from the lowest concentration 
to the highest concentration. In other words, mortality increased gradually with the increase in concentration of the test samples. The ethanolic extract of China Rose flowers showed almost no toxicity at all. This is reflected by the large value of $\mathrm{LC}_{50}$ of crude extract $(2332 \mu \mathrm{g} / \mathrm{ml})$ which was obtained by extrapolation of the percentage mortality vs logarithmic concentration curve. Hence, the extract of $H$. rosasinensis flowers has the potential to be used as edible food color.

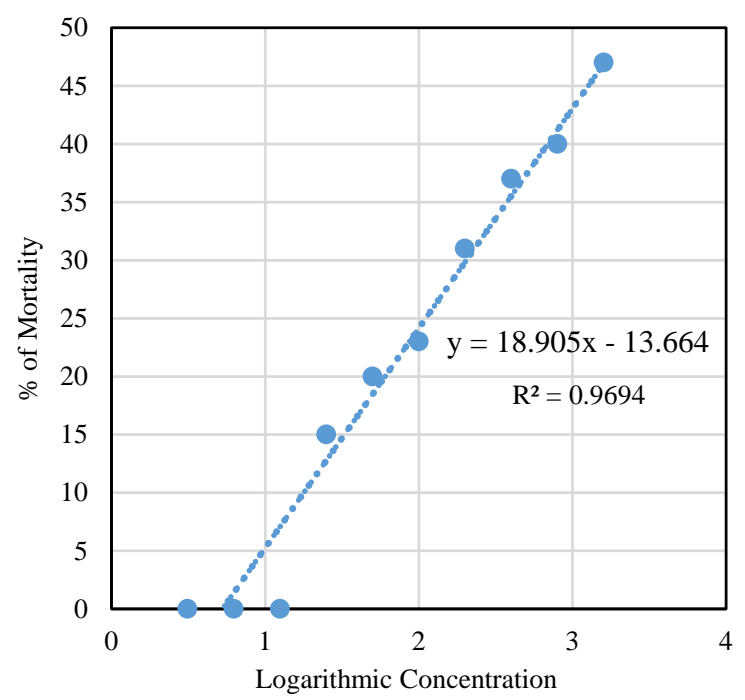

Fig. 4: Effect of sample concentration on mortality

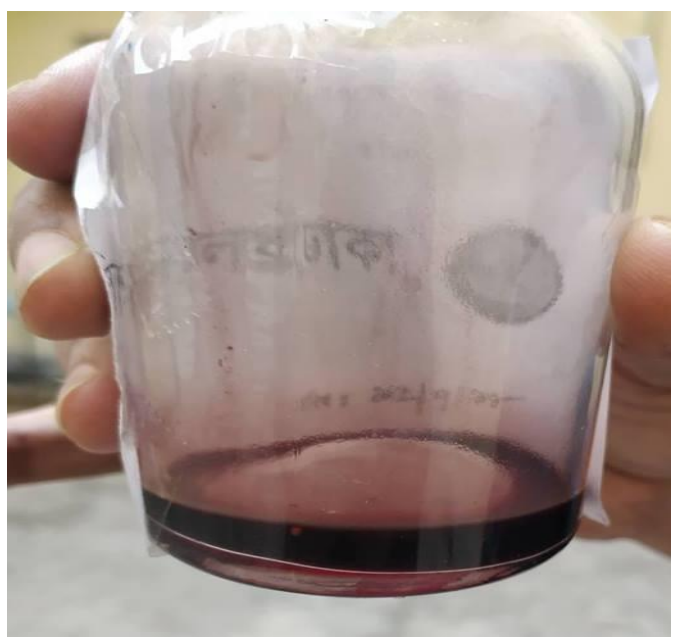

Fig. 5: Red coloured crude anthocyanin extract from H. rosasinensis flower

\section{Conclusions}

The study has successfully drawn up several conclusions which includes:

a. Extraction of crude anthocyanin is more efficient by Soxhlet extraction when the ratio of mass of dried flowers and volume of ethanol used was 1:20, at which the recovery of crude anthocyanin extract was $3.75 \%$. b. The $H$. rosasinensis flower extract were subjected to Brine Shrimp Lethality Test for preliminary screening of toxicity. The very low cytotoxicity ( $\mathrm{LC}_{50}$ value $2332 \mu \mathrm{g} / \mathrm{ml}$ ) exhibited by the crude extract indicates the absence of toxic compounds.

The results of this research shows the possibility of using crude extract of $H$. rosasinensis flower as a feedstock for natural colorant to food.

The following aspects can be evaluated in future research:

a. Evaluation of the change in extraction efficiency by altering the parameters such as particle size of the solute, nature of solvent, time of operation, etc. and selecting the optimum extraction condition.

b. Conducting acute toxicity test and chronic toxicity test for being completely sure about the nontoxic nature of the color pigments before commercial production.

c. Study the effect of change of temperature, $\mathrm{pH}$ and light on the stability of flower extract.

d. Determination of the shelf-life of the crude color.

e. Developing a method for large-scale commercial production of food color.

\section{Acknowledgements}

Supports from Department of Chemical Engineering in Bangladesh University of Engineering and Technology, and Bangladesh Council of Scientific and Industrial Research are gratefully acknowledged.

\section{References}

[1] Montaser M. M., Alkafafy M. E. (2013), Effects of Synthetic Food Color (Carmoisine) on Expression of Some Fuel Metabolism Genes in Liver of Male Albino Rats, Life Science Journal, 22013(10)

[2] Bride P., Timberlake C. F. (1997), Food Chemistry, 58, pp.103-109

[3] https://www.ncbi.nlm.nih.gov/pmc/articles/PMC5613 902/\#CIT0001

[4] Cho M. J., Howard L. R., Prior R. L. (2004), Flavonoid glycosides and antioxidant capacity of various blackberry, blueberry and red grape genotypes determined by high-performance liquid chromatography/mass spectrometry. Journal Science Food and Agriculture, pp.1771-1782

[5] Rodriguez-Saona L. E., Wrolstad R. E. (2001), Extraction, Isolation, and Purification of Anthocyanins. Current Protocols in Food Analytical Chemistry, 00(1), F1.1.1-F1.1.11

[6] Luque de Castro M. D., García-Ayuso L. E., (2000), Encyclopedia of Separation Science

[7] Ghafoor K., Choi Y. H., Jeon J. Y. and Jo I. H., (2009), Optimization of ultrasound-assisted extraction of phenolic compounds, antioxidants, and anthocyanins from grape (Vitis vinifera) seeds. Journal of 
Agriculture and Food Chemistry, 57: 4988-4994

[8] Ashitha G. N., Prince M. V., Sanjay P. (2020), Microwave assisted extraction of anthocyanin from Hibiscus rosa-sinensis. Journal of Pharmacognosy and Phytochemistry, 9 (2), pp. 1418-1424

[9] Ramprasath R., Kavi G. G., Rathi T. S. (2017), Isolation of Natural Dyes from Hibiscus Rosa Sinensis and Marigold Flower and Dyeing Properties of the Dyes On Cotton Cloth, IOSR Journal of Applied Chemistry (IOSR-JAC), 10(5), PP 74-79
[10] Aryanti, N., Nafiunisa, A. and Wardhani, D. H. (2019), Conventional and ultrasound-assisted extraction of anthocyanin from red and purple roselle (Hibiscus sabdariffa L.) calyces and characterization of its anthocyanin powder, International Food Research Journal, 26(2), pp.529-535

[11] Meyer B. N., Ferrigni N. R., Putnam J. E., Jacobsen L. B., Nichols D. E., McLaughlin J. L.. (1982), Brine shrimp: a convenient general bioassay for active plant constituents, Planta Medica, 45, pp. 31-34

(C)Bangladesh Uni. of Engg. \& Tech. 